\title{
Síndrome vasopléjico en cirugía cardíaca
}

\author{
Dr. Rafael Mendiburu
}

\section{Resumen}

El síndrome de respuesta inflamatoria sistémica constituye un conjunto de situaciones clínicas de diferente gravedad, que se presenta con una incidencia de hasta el 25\% durante las primeras horas del posoperatorio de cirugía cardíaca. En esta revisión, se analizan sus aspectos fisiopatológicos y los factores de riesgo que favorecen su desarrollo. Se plantea un enfoque diagnóstico, con la presentación final de una propuesta terapéutica.

Palabras clave: $\quad$ CIRUGÍA CARDÍACA

POSOPERATORIO

VASOPLEJIA

VASOCONSTRICTORES

CORTICOIDES

\section{Vasoplegia syndrome in cardiac surgery}

\section{Summary}

The systemic inflammatory response syndrome constitutes a set of clinical situations of different severity, which presents with an incidence of up to $25 \%$ during the first hours after cardiac surgery. In this review, the pathophysiological aspects and the risk factors that favor its development are analyzed. A diagnostic approach is proposed, with the final presentation of a therapeutic proposal.

Key words: $\quad$ CARDIAC SURGERY

POSTOPERATIVE

VASOPLEGIA

VASOCONSTRICTORS

CORTICOSTEROIDS

\section{Síndrome de vasoplegia em cirurgia cardíaca}

\section{Resumo}

A síndrome da resposta inflamatória sistêmica constitui um conjunto de situações clínicas de gravidade diversa, que se apresenta com incidência de até $25 \%$ nas primeiras horas de pós-operatório de cirurgia cardíaca. Nesta revisão, são analisados os aspectos fisiopatológicos e os fatores de risco que favorecem o seu desenvolvimento. É proposta uma abordagem diagnóstica, com a apresentação final de uma proposta terapêutica.

Palavras chave: CIRURGIA CARDÍACA

PÓS-OPERATÓRIO

VASOPLEGIA

VASOCONSTRITORES

CORTICOSTEROIDES

\section{Introducción}

El síndrome de respuesta inflamatoria sistémica (SIRS) es un continuo que en un extremo presenta una reacción leve, casi inadvertida (en la mayoría de los casos), y en el otro un shock vasopléjico con falla multiorgánica. Una proporción importante de los casos de SIRS posoperatorios se presenta como sín- drome vasopléjico (SV), que es el tema que motiva este trabajo.

La frecuencia del SV oscila de acuerdo a las diferentes series entre $5 \%-25 \%$; es una forma severa de SIRS, donde la vasodilatación se torna refractaria al manejo convencional y se extiende más allá de las primeras $6 \mathrm{~h}$ del posoperatorio inmediato (POI). Si 
esta situación se perpetúa por $36-48$ h, la mortalidad aumenta de $16 \%$ a $27 \%$. El SV es una condición potencialmente reversible si se inicia un tratamiento óptimo antes de que se instaure un estado de shock refractario. Esta ventana terapéutica parece extenderse hasta el primer día del posoperatorio.

\section{Definición}

Se define al SV como un estado de hipoperfusión tisular causado por vasodilatación refractaria al uso de vasopresores. Se manifiesta clínicamente como hipotensión arterial en presencia de gasto cardíaco (GC) normal (1). En el posoperatorio de cirugía cardíaca (POCC) utilizaremos los siguientes parámetros:

- Presión arterial sistólica $<80 \mathrm{mmHg}$.

- Presión venosa central (PVC) $<5 \mathrm{mmHg}$.

- Índice cardíaco (IC) $>2,2 \mathrm{l} / \mathrm{min} / \mathrm{m}^{2}$.

- Presión de oclusión de la arteria pulmonar (POAP) $<10 \mathrm{mmHg}$.

- Resistencias vasculares sistémicas (RVS) $<800$ $\operatorname{dinas} / \mathrm{s} / \mathrm{cm}^{-5}$.

- Bajo apoyo de noradrenalina (NA) >0,5 ga$\mathrm{mas} / \mathrm{kg} / \mathrm{min}$.

Algunos autores incluyen un aumento de ácido láctico y la presencia de oliguria ${ }^{(2)}$. El punto de mayor consenso es la constatación de RVS bajas, a pesar de que en la mayoría de los pacientes no obtenemos los datos del catéter de arteria pulmonar (CAP), prácticamente en desuso, pero de gran utilidad en este caso.

El SIRS, que es el paso previo y más benigno de este cuadro grave, se define por tener dos o más de los criterios de las Conferencias de Consenso de 1991 y $2001^{(3,4)}$ :

- Temperatura $>38^{\circ} \mathrm{Co}<36^{\circ} \mathrm{C}$.

- Hiperleucocitosis $>12.000 / \mathrm{mm}^{3}$ o leucopenia < $4.000 / \mathrm{mm}^{3}$.

- Taquicardia $>100 \mathrm{cpm}$.

- Taquipnea $>20$ rpm o presión arterial de $\mathrm{CO}_{2}$ $<32 \mathrm{mmHg}$.

Estos criterios pasan inadvertidos por ser habituales en el POCC, y por corregirse fácilmente con aporte de volumen y con dosis bajas de vasopresores.

\section{Fisiopatología}

La lesión endotelial provoca dilatación arteriolar y pérdida de la autorregulación, que son los causantes del SV. Durante la circulación extracorpórea (CEC) se generan alteraciones en los mecanismos homeostáticos, que dan como resultado una res- puesta inflamatoria sistémica. Esta respuesta inflamatoria se divide en dos fases fundamentales: temprana y tardía. La fase temprana, que consiste en la activación del sistema de contacto, con la activación simultánea del sistema de coagulación y del complemento, y el consiguiente aumento de la fibrinólisis. A nivel celular hay activación de linfocitos, monocitos, neutrófilos, plaquetas y células endoteliales. La degranulación mastocitaria conlleva a una liberación de citocinas proinflamatorias, tales como la interleucina (IL) 4, IL 10, interferón gamma y factor de necrosis tumoral alfa. La fase tardía de la respuesta inflamatoria a la CEC se produce por isquemia y reperfusión ocasionadas por el despinzamiento aórtico, y el reclutamiento de lechos vasculares previamente no perfundidos. Esto conduce a daño endotelial mediante la activación y la amplificación de la respuesta humoral del sistema de complemento, y la liberación de radicales libres de oxígeno. El óxido nítrico (NO) también está estrechamente relacionado con el desarrollo del SV. La producción de NO se da a través de la activación de dos tipos de NO sintetasa (NOS): una constitutiva endotelial (eNOS) y una inducible (iNOS). El NO activa la guanilatociclasa para iniciar la producción de cGMP, que inhibe la actividad contráctil de la musculatura lisa vascular, causando vasodilatación. En la fisiopatología del SV se han propuesto mecanismos subyacentes independientes del NO, tales como la bradicinina, un péptido vasodilatador cuya degradación es inhibida por los inhibidores de la enzima convertidora de angiotensina (IECA), la hipotermia y la CEC.

Esto explica la resistencia a las catecolaminas que caracteriza al SV. Finalmente, se han planteado también factores hormonales, ya que algunos estudios han mostrado deficiencia absoluta o relativa de hormonas involucradas en la regulación de la presión arterial, tales como vasopresina, tiroxina y cortisol. Clásicamente, el SV se ha asociado a pacientes tratados mediante cirugía cardíaca con CEC. Sin embargo, se ha observado una incidencia del 2,8\% en cirugía cardíaca de revascularización miocárdica sin CEC, lo cual apunta a la existencia de otros mecanismos desencadenantes. En virtud de esto, es probable que el SV sea una vía final común en la cual múltiples factores confluyen en una lesión endotelial promotora de mecanismos vasodilatadores con pérdida de la autorregulación ${ }^{(1)}$.

\section{Factores de riesgo}

La CEC, al generar una respuesta inflamatoria sistémica por los mecanismos discutidos en la sección previa, es el principal factor de riesgo para el SV.Su 
duración, la presencia de hipotensión y la temperatura durante la misma pueden propiciar el desarrollo de SV. Se ha determinado que el tiempo de CEC $>100 \mathrm{~min}$, una presión arterial media $(\mathrm{PAM})<50$ mmHg y una temperatura $<34^{\circ} \mathrm{C}$, se asocian a la aparición de SV. Con respecto a esto, los nuevos filtros usados en la CEC aislarían el pasaje de mediadores a la circulación general, atenuando esta posibilidad, pero no eliminándola. El SV aparece en los pacientes con un mayor puntaje de EuroSCORE y con menor fracción de eyección del ventrículo izquierdo. Con respecto a los fármacos vasodilatadores, la administración de IECA en las $24 \mathrm{~h}$ previas al procedimiento es un factor de riesgo establecido con una incidencia de SV de $26,9 \%$. No solamente los medicamentos vasodilatadores están implicados en el desarrollo del SV, sino también la dependencia de inotrópicos en el preoperatorio, presumiblemente por regulación a la baja de los receptores adrenérgicos. El tipo de cirugía y su indicación desempeñan un papel importante, encontrándose una mayor frecuencia en cirugía con apertura de cavidades que en la cirugía de revascularización coronaria. En esta última, es más frecuente en procedimientos realizados con CEC. Con respecto a la indicación de la cirugía, está establecido que pacientes tratados mediante cirugía valvular por endocarditis infecciosa están en mayor riesgo de SV en comparación con pacientes con otras indicaciones. La cirugía para insuficiencia cardíaca terminal presenta la cohorte de pacientes con mayor incidencia de SV.

\section{Diagnóstico}

El SV es un diagnóstico de exclusión en el cual se debe tener un alto índice de sospecha, teniendo en cuenta los factores predisponentes. El enfoque diagnóstico debe ser adecuado a las diferentes etapas del perioperatorio, descartando los diagnósticos diferenciales de acuerdo con dichas etapas. Durante la CEC el signo más temprano es la PAM persistentemente baja a pesar de un aporte de fluidos adecuado (figura 1).

Salida de CEC: durante esta fase el SV simula un déficit de volumen. Los hallazgos hemodinámicos son una PAM baja, acompañada de PVC y POAP bajas. El punto clave para diferenciar el SV de una hipovolemia es la medición del GC: en el caso de hipovolemia va a estar bajo, mientras que por el contrario en el shock o SV se encuentra incrementado.

La medición del GC por termodilución con el CAP continúa siendo el método de referencia, pero su uso ha declinado en las últimas décadas a favor de la utilización rutinaria de la ecocardiografía transesofágica intraoperatoria. Ante la sospecha de
SV es necesario descartar alteraciones como trastornos regionales de la contractilidad, disfunción valvular y disfunción aguda del ventrículo derecho, los cuales podrían requerir retorno a la CEC. En el PO la valoración por ecocardiograma transtorácico puede ser suficiente si la ventana ecocardiográfica es adecuada. El GC calculado con el modo Doppler pulsado, utilizando las proyecciones transgástricas de eje largo o cinco cámaras, se encuentra aumentado a diferencia del déficit volémico, en el que el GC estará deprimido. Cuando se evalúa el paciente con el tórax cerrado es posible encontrar variación exagerada en el colapso de la vena cava inferior (VCI) y de la integral de la velocidad en función del tiempo aórtica.

\section{Tratamiento}

Luego del manejo de la precarga con el adecuado volumen (aporte de cristaloides), el tratamiento se centra en los vasoconstrictores, que se pueden dividir para su análisis en: catecolaminas, hormonas e inhibidores del $\mathrm{NO}^{(5)}$.

\section{Catecolaminas}

Fármacos que estimulan los receptores adrenérgicos para activar el canal $\mathrm{L}$ de calcio, produciendo aumento del calcio citosólico con la consecuente contracción de la fibra muscular lisa vascular.

NA: el incremento de la presión arterial se da por estimulación de los receptores alfa 1 adrenérgicos de la musculatura lisa vascular y del receptor B1 a nivel renal y cardíaco.

Fenilefrina: es un agonista puro de los receptores alfa 1 adrenérgicos. Sus efectos hemodinámicos se limitan al aumento de la PAM con pocos efectos sobre los otros parámetros.

Adrenalina: Es un potente agonista alfa y beta adrenérgico, que ejerce su acción presora por un mecanismo dual de vasoconstricción y aumento del GC.

Los efectos adversos de las catecolaminas son comunes a todas ellas ya que comparten mecanismos de acción. La sobrecarga citosólica de calcio es la explicación de dichos efectos pues favorece la disfunción diastólica, la taquicardia, el desarrollo de arritmias y la hipoperfusión intestinal, renal y cutánea.

\section{Hormonas}

La vasopresina, terlipresina (profármaco de la anterior) y la angiotensina II funcionan por mecanismos independientes a los receptores adrenérgicos.

Vasopresina: explorada en el estudio VASST ${ }^{(6)}$ y VANISH $^{(7)}$ (sepsis severa y shock séptico) y el 


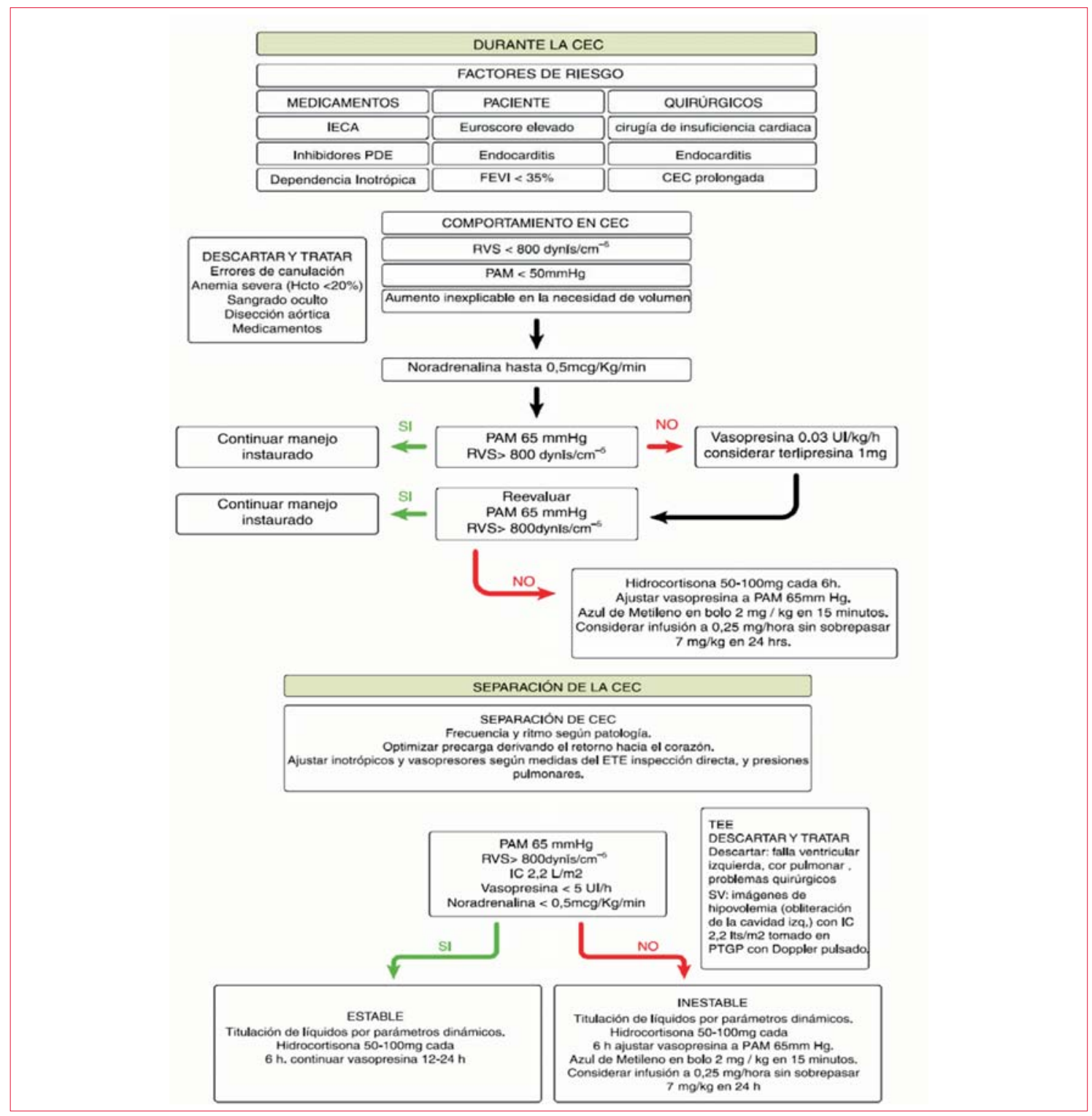

Figura 1. Algoritmo propuesto de manejo para el SV. TEE: ecocardiograma transesofágico; FEVI: fracción de eyección del ventrículo izquierdo; CEC: circulación extracorpórea; HCTO: hematocrito; IECA: inhibidores de la enzima conversora de angiotensina; PAM: presión arterial media; PDE: fosfodiesterasa; PTGP: plano transgástrico profundo; RVS: resistencias vasculares sistémicas; IC: índice cardíaco; SV: síndrome vasopléjico ${ }^{(1)}$.

$\operatorname{VANCS}^{(8)}$ (POCC), tiene acciones multisistémicas tales como estímulo de la gluconeogénesis, agregación plaquetaria y liberación de adrenocorticotropina. Produce sus efectos a través de los receptores V1 y V2. Usada como rescate en el shock refractario a catecolaminas, ofrece mayores beneficios en comparación con dosis altas de NA. En resumen, los efectos hemodinámicos de la vasopresina en SV consisten en el aumento de la PAM sin efectos sobre el IC y una modesta disminución de la frecuencia cardíaca y de la incidencia de arritmias. También se ha evidenciado una importante reducción en la necesidad de NA. Esta medicación se encontrará disponible en nuestro medio a partir de este año. Con el nombre comercial de Novopressina $\mathrm{V}$, cada ampolla de $1 \mathrm{ml}$ tiene $20 \mathrm{UI}$; la dosis recomendada para este uso es de $0,03 \mathrm{UI} / \mathrm{kg} / \mathrm{h}$ o $0,01-0,04 \mathrm{UI} / \mathrm{min}$.

Terlipresina: presenta el mismo perfil hemodinámico de la vasopresina. Sin embargo exhibe agonismo selectivo sobre los receptores V2, por lo cual produce vasoconstricción pura en todos los lechos. Puede generar un aumento de la incidencia de injuria renal aguda. Otro aspecto problemático es su vida media prolongada, y su forma de dosificación tampoco es clara. No está aprobada para este uso por FDA en Estados Unidos y Canadá; su uso sí está aprobado en Europa, Australia y Nueva Zelanda. Esta droga está contraindicada cuando hay hiper- 


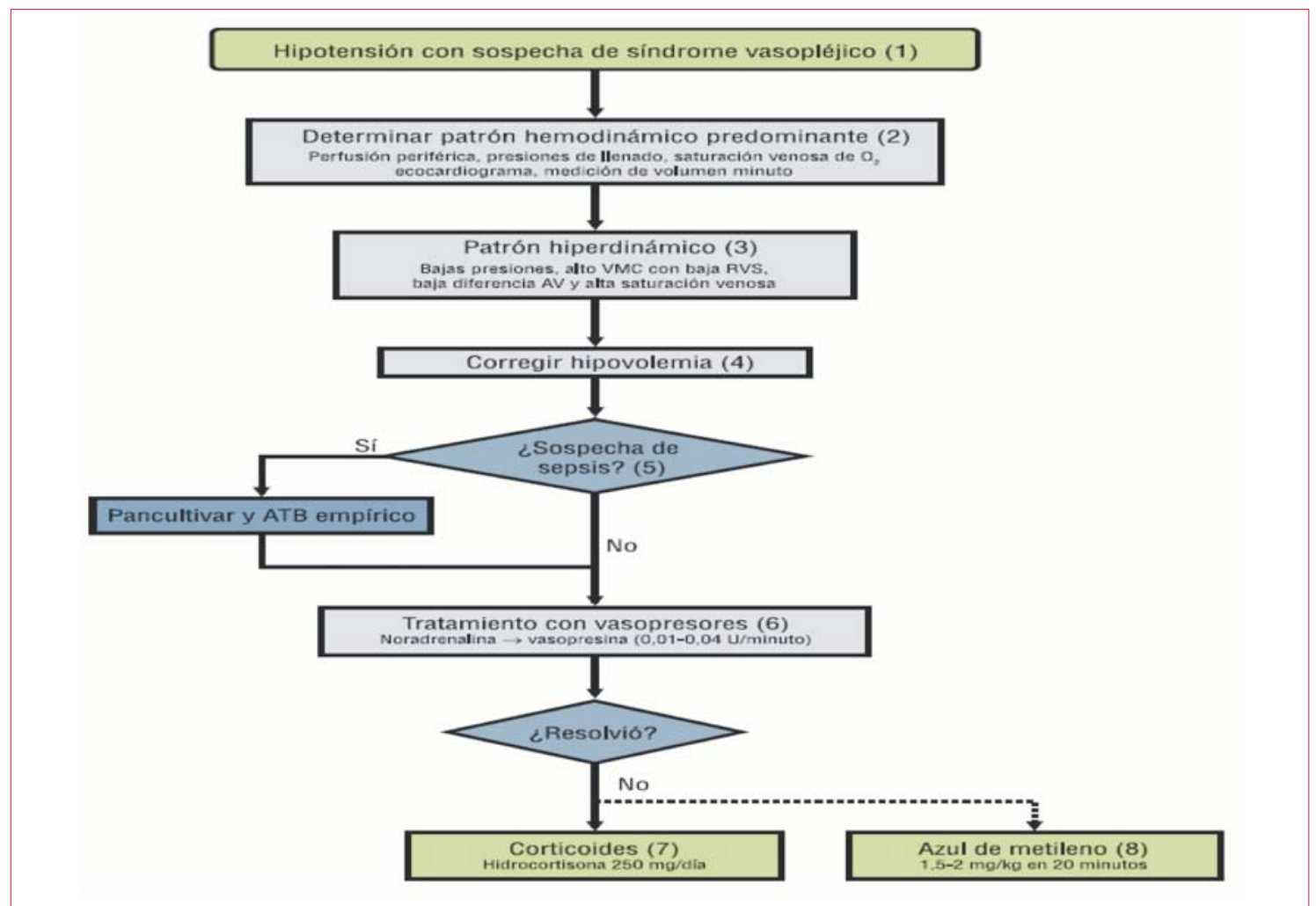

Figura 2. Shock vasopléjico. Algoritmo de tratamiento considerando la posible etiología séptica. ATB: antibiótico; AV: arteriovenosa; RVS: resistencia vascular sistémica; VMC: volumen minuto cardíaco(7).

sensibilidad a la misma, asma severa o historia de ataque cerebrovascular.

Angiotensina II: Su uso en cirugía cardíaca se ha realizado solo en forma anecdótica en pacientes con SV refractario a catecolaminas recibiendo IECA y amiodarona.

\section{Inhibidores del óxido nítrico}

Azul de metileno: su utilización en estados de vasoplejia resistente a vasopresores resulta una alternativa útil en el contexto de cirugía cardíaca. Posee un potente efecto inhibitorio directo sobre la eNOS y sobre la iNOS (menos potente sobre esta última) y bloquea la formación de cGMP (segundo mensajero implicado en la regulación de la contracción del músculo liso) al disminuir la acción de la enzima guanilatociclasa. Estos efectos son mediados a través de la oxidación del hierro. Cabe resaltar que el beneficio de este fármaco es mayor cuando se utiliza precozmente. Se han propuesto múltiples esquemas terapéuticos para el manejo del SV en cirugía cardíaca, siendo la dosis más común 1 a $2 \mathrm{mg} / \mathrm{kg}$ durante 10 a 30 min por vía intravenosa. En nuestro medio se encuentran disponibles para este uso ampollas de $1 \mathrm{mg}$. Los efectos adversos no son comunes, pero pueden ser serios. Los efectos secundarios son dependientes de la dosis siendo los más frecuen- tes: digestivos altos, coloración de la orina, aumento de las presiones pulmonares, interferencia con lecturas de oximetría de pulso, arritmias graves. Se recomienda no sobrepasar de $5-7 \mathrm{mg} / \mathrm{kg}$ por día. Es un inhibidor de la monoaminooxidasa, por lo cual se debe administrar con precaución en pacientes que estén recibiendo inhibidores de la recaptación de serotonina. En pacientes con insuficiencia renal puede haber acumulación de sus metabolitos, por lo que se debe ajustar la dosis. Otros efectos descritos son el coma y la metahemoglobinemia.

\section{Esquema terapéutico}

De especial interés resulta el hecho de que la combinación de un segundo vasopresor (vasopresina) con mecanismo de acción distinto, es superior a la utilización de monoterapia a dosis alta, y que el uso de sustancias vasoactivas en SV en el POCC, no se asocia a hipoperfusión visceral. El manejo inicial con aminas vasoactivas sigue siendo el tratamiento de primera línea. La NA en monoterapia a dosis elevadas tiene dos desventajas: por un lado, poca respuesta por la inactivación del canal del calcio típica del SV y por otro la sobrecarga miocárdica de calcio que ocasiona disfunción diastólica, arritmias, y regulación a la baja de los receptores adrenérgicos. Cuando las dosis de NA alcanzan valores superiores 
a 0,75 gamas $/ \mathrm{kg} / \mathrm{min}$ la mortalidad puede llegar hasta el $86 \%$ en pacientes con disfunción orgánica múltiple, por lo que se recomienda el uso concomitante de otro vasopresor con mecanismos de acción alternativos. La vasopresina tiene evidencia de alta calidad para su utilización y el azul de metileno se reserva un papel coadyuvante o de rescate ${ }^{(5)}$.

Como medidas preventivas de este síndrome se recomienda la suspensión de los IECA $24 \mathrm{~h}$ antes de la cirugía, así como la utilización de filtros para mediadores en la CEC.

Una vez que el cuadro se ha instalado, se propone el siguiente algoritmo terapeútico: la NA es el vasopresor de primera línea. El objetivo es lograr una PAM de $65 \mathrm{mmHg}$. Si es necesaria una dosis de NA superior a 0,5 gamas $/ \mathrm{kg} / \mathrm{min}$, se recomienda el inicio de vasopresina en infusión continua. La terlipresina, en bolo de $1 \mathrm{mg}$, es una alternativa, aunque se requiere mayor evidencia para poder recomendar su uso. El azul de metileno se reserva para aquellos casos en los que se requiere una dosis de NA superior a 0,5 gamas $/ \mathrm{kg} / \mathrm{min}$, junto a dosis altas de vasopresina, para mantener la PAM objetivo. En este escenario, se administra un bolo de azul de metileno de $2 \mathrm{mg} / \mathrm{kg}$ como terapia de rescate, lográndose, en la mayoría de los pacientes, un aumento significativo de las RVS, y un descenso significativo del soporte hemodinámico. En caso de persistencia de la situación de vasoplejia, se procederá a iniciar una perfusión de azul de metileno a $0,25 \mathrm{mg} / \mathrm{kg} / \mathrm{h}$. En este contexto, podría ser razonable la administración de hidrocortisona de 50-100 mg cada $6 \mathrm{~h}$ (deficiencia absoluta o relativa de cortisol). En pacientes con riesgo de síndrome serotoninérgico, se considera a la hidroxicobalamina como una alternativa al azul de metileno ${ }^{(9)}$. Además de este manejo inicial en base a volumen, vasopresores, azul de metileno y corticoides, frente a un cuadro de shock distributivo es pertinente realizar una ronda de cultivos, e iniciar un tratamiento antibiótico empírico. La sospecha de infección será más alta en aquellos pacientes que estuvieron internados previamente, o cuando el estado inflamatorio comienza varios días después de la cirugía. Esto se refleja en el segundo algoritmo de tratamiento representado en la figura $2^{(10)}$.

\section{Rafael Mendiburu,}

https://orcid.org/000-0003-1669-6770

Este artículo fue aceptado para su publicación por: Editor invitado Dr. Oscar Bazzino

\section{Bibliografía}

1. Orozco DM, Triana Schoonewolff CA, Orozco Vinasco AC. Síndrome vasopléjico en cirugía cardíaca: definición, fisiopatología, enfoque diagnóstico y manejo. Revista Española de Anestesiología y Reanimación. 2019;66(5):277-287. doi: 10.1016/j.redar. 2018.12.011

2. Synger M, Deutschman CS, Seymour CW, Shankar-Hari M, Annane D, Bauer Met, et al. The third international consensus definitions for sepsis and septic shock (Septic-3). JAMA. 2016; 315 (8): 801-10. doi: 10.1001/jama.2016.0287

3. Members of the American College of Chest Physicians/Society of Critical Care Medicine Consensus Conference Committee. Definitions for sepsis and organ failure and guidelines for the use of innovative therapies in sepsis. Crit Care Med. 1992; 20(6): 864-74.

4. Levy MM, Fink MP, Marshall JC, Abraham E, Angus D, Cook D, et al. 2001 SCCM/ESICM/ ACCP/ATS/SIS International sepsis definitions conference. Intensive Care Med. 2003; 29(4):530-8. doi: $10.1007 / \mathrm{s} 00134-003-1662-\mathrm{x}$

5. Ortoleva J, Shapeton A, Vanneman M, Dalia A. Vasoplegia during Cardiopulmonary Bypass: $\mathrm{Cu}-$ rrent Literature and Rescue Therapy Options. J Cardiothorac Vasc Anesth. 2019; 34(10):1-10. doi: 10.1053/j.jvca.2019.12.013

6. Russell JA, Walley KR, Singer J, Gordon AC, Hébert PC, Cooper DJ, et al; for the VASST Investigators. Vasopressin vs norepinephrine infusion in patients with septic shock. N Engl J Med. 2008; 358: 877-87. doi: 10.1056/NEJMoa067373

7. Gordon AC, Mason AJ, Thirunavukkarasu N, Perkins GD, Cecconi M, Cepkova M, et al. for the VANISH Investigators. Effect of early vasopressin vs. norepinephrine on kidney failure in patients with septic shock. JAMA 2016; 316(5): 509-18. doi:10.1001/jama.2016.10485

8. Abrahao Hajjar L, Vincent JL, Barbosa Gomes Galas FR, Rhodes A, Landoni G, Atsushi Osawa ER, et al. Vasopressin vs norepinephrine in patients with Vasoplegic shock after cardiac surgery. The VANCS randomized controlled trial. Anesthesiology 2017; 126(1): 85-93. doi: 10.1097/ALN.0000000000 001434

9. Furnish C, Mueller SW, Kiser TH, Dufficy L, Sullivan B, Beyer JT. Hydroxicobalamin versus methylene blue for vasoplegic syndrome in cardiothoracic surgery: a retrospective cohort. J Cardiothorac Vasc Anesth. 2020; 1-8. 34(7):1763-70. doi: 10.1053/j.jvca.2020.01.033

10. Benzadón M. Algoritmos en el posoperatorio de cirugía cardiovascular. Buenos Aires: Inter-Médica; 2012. 